\title{
An integrative approach for building personalized gene regulatory networks for precision medicine
}

\author{
Monique G. P. van der Wijst ${ }^{\dagger}$ BD, Dylan H. de Vries ${ }^{\dagger}$, Harm Brugge, Harm-Jan Westra and Lude Franke*
}

\begin{abstract}
Only a small fraction of patients respond to the drug prescribed to treat their disease, which means that most are at risk of unnecessary exposure to side effects through ineffective drugs. This inter-individual variation in drug response is driven by differences in gene interactions caused by each patient's genetic background, environmental exposures, and the proportions of specific cell types involved in disease. These gene interactions can now be captured by building gene regulatory networks, by taking advantage of RNA velocity (the time derivative of the gene expression state), the ability to study hundreds of thousands of cells simultaneously, and the falling price of single-cell sequencing. Here, we propose an integrative approach that leverages these recent advances in single-cell data with the sensitivity of bulk data to enable the reconstruction of personalized, cell-type- and context-specific gene regulatory networks. We expect this approach will allow the prioritization of key driver genes for specific diseases and will provide knowledge that opens new avenues towards improved personalized healthcare.
\end{abstract}

\section{Background}

In the past decade, genome-wide association studies (GWAS; Box 1) have identified over 10,000 genetic risk factors, mainly single nucleotide polymorphisms (SNPs), for more than 100 common diseases [1]. Together these GWAS loci can explain up to $25 \%$ of the heritability of complex diseases [2] and up to $56 \%$ of disease-related

\footnotetext{
* Correspondence: lude@ludesign.nl

${ }^{\dagger}$ Monique G. P. van der Wijst and Dylan H. de Vries contributed equally to this work.

Department of Genetics, 5th floor ERIBA building, Antonius Deusinglaan 1, 9713AV Groningen, University of Groningen, University Medical Center Groningen, Groningen, The Netherlands
}

traits [3]. The majority of these genetic risk factors are located in non-coding regions [4] and, as the function of these regions is challenging to decipher, it remains largely unclear how the SNPs are linked to disease. Several studies have shown that the gene nearest to the genetic association may not always be the causal gene [5-7]. Consequently, more sophisticated approaches have been developed to unravel the link between genetic risk factors and disease (for example, by identifying the disease-causing cell types, genes, and pathways; Fig. 1). Expression quantitative trait loci (eQTL) studies, for example, have been performed to identify the local (cis-eQTL) and distal (trans-eQTL) downstream effects of genetic variation on gene expression $[8,9]$. These eQTL studies have provided the first clues about how genetic variation is linked to disease (Fig. 2a). Other methods to further prioritize putatively causal genes include co-localization analysis, fine-mapping, and summary-data-based Mendelian randomization (for detailed discussions of these techniques see $[10,11])$. To provide a greater understanding of gene regulatory mechanisms, several large consortia-including the ENCODE project [12], FANTOM [13], Epigenome Roadmap [14], and Blueprint [15] - have systematically classified more than $80 \%$ of the genome as non-coding regulatory elements. Genetic variation has now been linked to many of these elements, including epigenetic marks $[16,17]$, transcription factor binding and chromatin accessibility [18, $19]$, and post-transcriptional regulation [20, 21].

Studies to date have emphasized the importance of studying both gene expression [22] and its regulation. However, despite these advances in our understanding of GWAS variants, a recent study of 7051 samples from 449 donors across 44 tissues from the Genotype-Tissue Expression (GTEx) project linked only $61.5 \%$ of the SNPs within a GWAS locus to an eQTL effect [23]. The reason that not all GWAS SNPs can be linked to an eQTL effect could be that eQTL studies have been performed in the wrong context for a specific disease. We

(c) The Author(s). 2018 Open Access This article is distributed under the terms of the Creative Commons Attribution 4.0 International License (http://creativecommons.org/licenses/by/4.0/), which permits unrestricted use, distribution, and reproduction in any medium, provided you give appropriate credit to the original author(s) and the source, provide a link to the Creative Commons license, and indicate if changes were made. The Creative Commons Public Domain Dedication waiver (http://creativecommons.org/publicdomain/zero/1.0/) applies to the data made available in this article, unless otherwise stated. 


\section{Box 1. Glossary}

Bayesian model: statistical modeling to calculate probabilities for an observation while taking into account the empirical or theoretical expected distribution of these observations or factors expected to influence the observations. Used in co-expression networks to assign probabilities for directionality between genes.

Benchmarking: comparing the performance of a computational model against a gold standard or known solution.

Co-expression network: an undirected network that describes which genes often behave in a coordinated manner. The network consists of nodes, representing genes, that are connected through edges that represent relationships between nodes. These relationships can be unweighted or weighted, indicating either a binary relationship (on/off) or a more continuous relationship.

Co-expression QTLS: SNPS that modulate the correlation between the co-expression of two genes. To identify these, many observations (for example, on multiple cells or tissues) per individual are required.

Co-localization: a method that determines whether the association signals in a locus correspond between two association studies (for example, between a GWAS and an eQTL study).

Context-dependent eQTLs: eQTLs for which the strength of association depends on a secondary factor. This may be either intrinsic (for example, expression of another gene or cell type frequency) or extrinsic (for example, environmental exposure). Gene expression data can be used as proxy measurements for both intrinsic and extrinsic factors.

Dropouts: genes that fail to be detected even though they are expressed (resulting in a zero-inflated gene expression distribution) due to incomplete mRNA capture by current scRNA-seq technologies.

Expression quantitative trait loci (eQTL): a SNP that explains a variation in gene expression levels. When the SNP explains the expression of a gene within a 1-megabase distance, it is called a cis-eQTL. When the SNP explains the expression of a gene beyond a 1-megabase distance, it is called a trans-eQTL.

Fine-mapping: a statistical approach that is used to prioritize the most likely causal genetic variant in a previously identified locus that is linked to a specific phenotype.

Gene regulatory network (GRN): a directional co-expression network that also contains information about the regulators that control gene expression.

Genome-wide association studies (GWAS): genome-wide approach in which genetic variants such as single nucleotide polymorphisms (SNPs) are linked to a molecular trait or disease.

Genotype by environment (GxE) interactions: interactions between an individual's genotype and the environment. Context-dependent eQTLS are a subset of GxE interactions.

Machine learning approaches: methods used to analyze massive amounts of data in order to build predictive models from multidimensional datasets.

Nyquist-Shannon sampling theorem: describes the sample frequency that is sufficient to capture all the information from a continuoustime signal of a finite bandwidth.

Precision medicine: healthcare that is individually tailored on the basis of a person's genetic, environmental, and lifestyle characteristics. Pseudotime: temporal sequences of gene expression states in cells inferred from measurements made at a single moment in time. RNA velocity: the rate of change of mRNA molecule abundances in the cell determined by modeling the relationship between unspliced mRNA (an indicator of current transcription) and spliced mRNA (an indicator of transcription in the recent past).

Simpson's paradox: a situation in which an observed relationship within different samples (or groups of data) disappears or reverses when the samples (or groups) are combined.

Summary-data-based Mendelian randomization (SMR): a summary statistics based variant of Mendelian randomization that leverages the principle that genetic variation is randomly assigned to a sample with a specific phenotype to infer causality between genetic variation and the phenotype in an observational study.

Unique molecular identifiers (UMIs): barcode sequences tagging individual molecules.

now know that many genetic risk factors have cell-type-specific effects [22, 24, 25] or are modulated by environmental factors $[26,27]$ and these are contexts that eQTL studies usually do not completely capture.
Independent genetic risk factors can converge into key regulatory pathways $[24,28]$ and may act beyond the disruption of individual genes [29, 30]. Therefore, we expect that a comprehensive overview of the many 


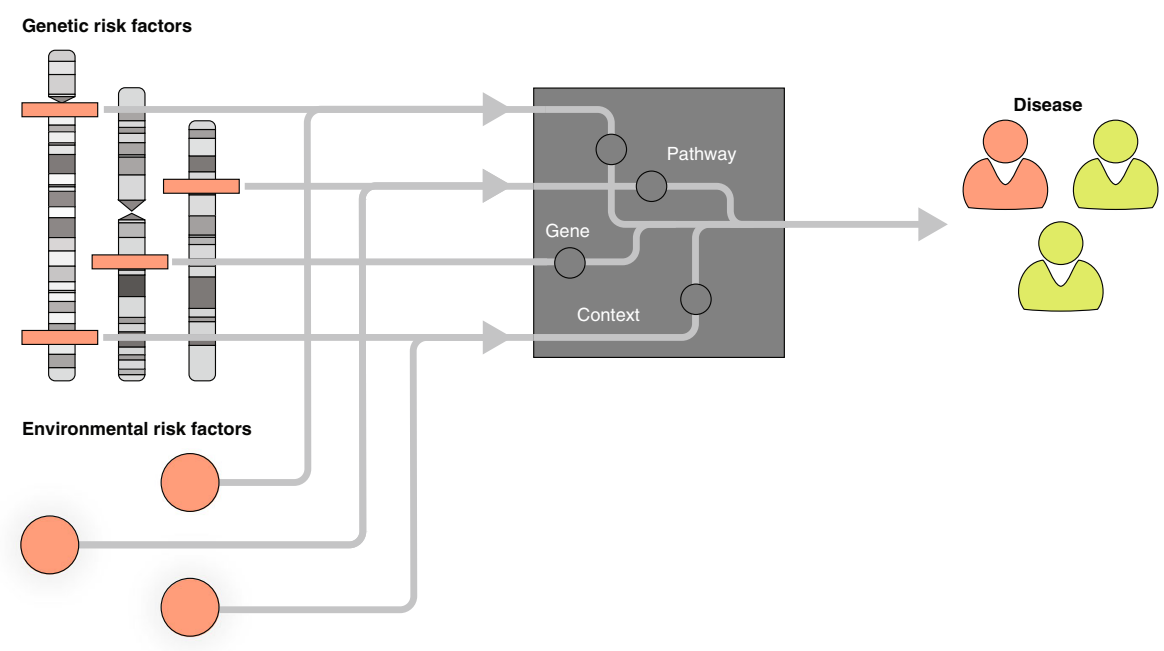

Fig. 1 The link between genetic and environmental risk factors in disease. Understanding the interplay between genetic and environmental risk factors enables identification of the disease-associated context, causal genes, and pathways. This leads to a better understanding of why certain individuals become ill, whereas others do not

processes at work will be required to better understand disease pathogenesis. This kind of overview can be acquired by reconstructing gene regulatory networks (GRNs) that are based on cell type [22, 24, 25], environment [26, 27], and an individual's genetic makeup [29, 30]. A GRN is a directional network of genes in which relationships between genes and their regulators are mapped. Understanding the effect of genetic variation on GRNs is particularly important because this may contribute to the large inter-individual variation in drug responsiveness (Fig. 3). At present, some of the most commonly prescribed drugs are effective in only 4 to $25 \%$ of the people for whom they are prescribed [31].

Here, we outline our vision for an integrative approach to reconstruct context-specific GRNs. We focus on gene expression-based regulatory networks because a wealth of gene expression data is already available and the generation of this type of data at the bulk and single-cell levels has advanced the most compared to other single-cell technologies. However, there are other molecular levels, such as metabolites or proteins, which should be included in GRNs in the future to capture the full complexity of a disease [32].

We begin with a brief introduction to the concept of a co-expression network and describe the methods used to create directional GRNs from co-expression networks using bulk data. We then discuss the limitations of bulk data and how these can be resolved by the unique properties of novel single-cell gene expression approaches to enable the reconstruction of causal GRNs. Methods used to reconstruct single-cell GRNs have been reviewed recently by Fiers et al. [33] and are therefore not covered in detail here. We conclude by describing how the combination of bulk and single-cell data can be used to reconstruct context-specific, personalized GRNs, and describe their use in advancing personalized healthcare.

\section{Gene networks in bulk data}

Understanding the pathways affected in disease requires a clear definition of which genes act together in specific cellular processes. To this end, model organisms have been instrumental in defining the most basic pathways present in each cell. By performing knockout experiments, for instance, the relationships between genes can be identified by studying the downstream effects on gene expression or enzymatic function, and these effects are now catalogued in databases such as KEGG [34] and REACTOME [35]. The pathways defined in these databases, however, can be incomplete or biased towards well-studied cellular phenotypes or genes. Co-expression networks and GRNs can therefore be used to extend the knowledge provided by such databases, and methods for constructing such networks have been reviewed in detail elsewhere [36, 37].

Gene networks can be used to infer the functions of unannotated genes by assuming that genes with similar functions are located close together in these networks (i.e. guilt-by-association) [38-42]. The clusters in the network can be overlapped with the genes that are affected by GWAS SNPs to identify the disease-associated pathways, using methods such as GRAIL [42], MAGENTA [43], and DEPICT [39] (Fig. 2b). However, knowing the functions of genes and the associations between genes is often insufficient to identify the key driver gene. 


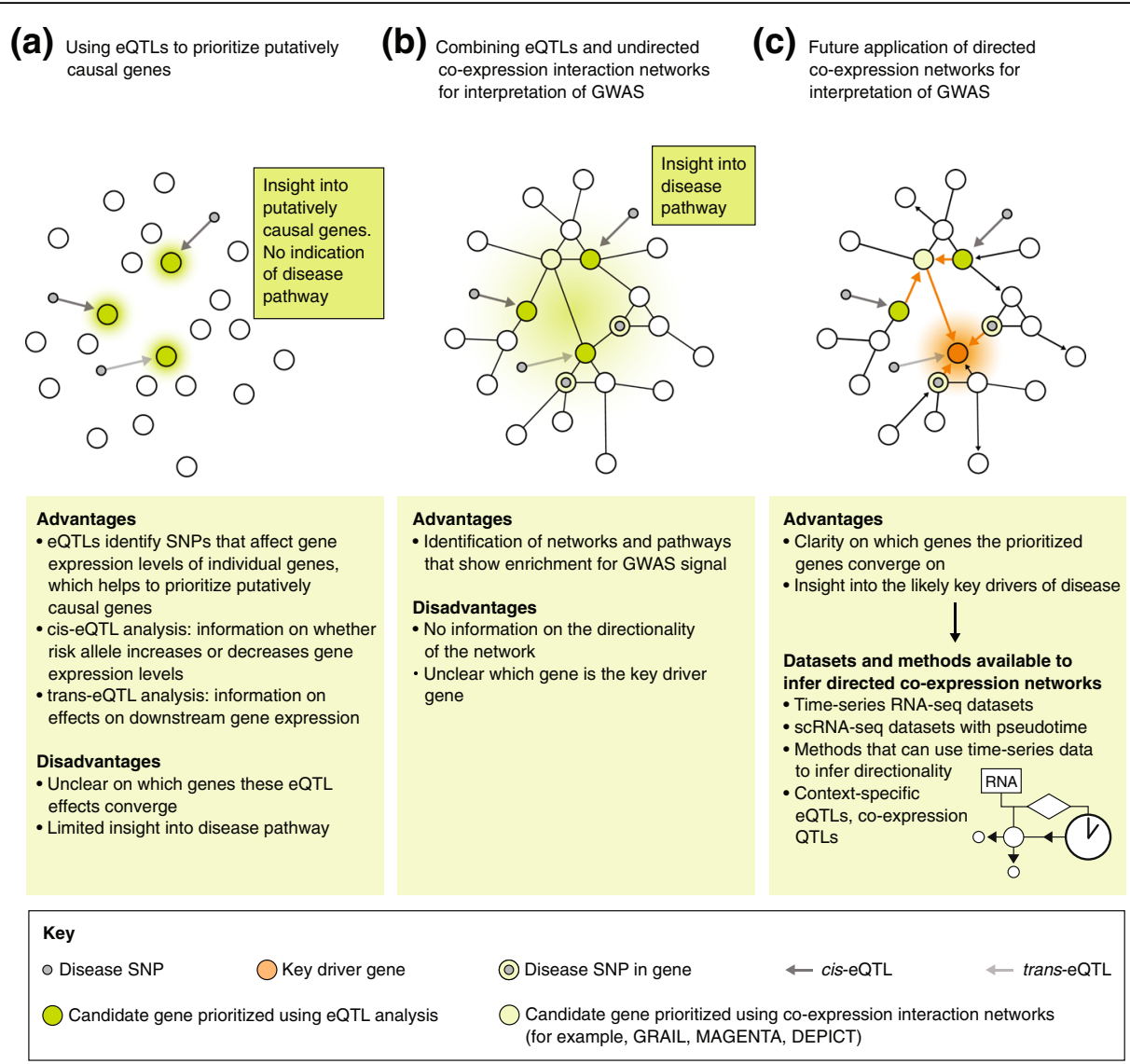

Fig. 2 Current and future approaches to understand the role of genetics in disease. a To identify putatively causal genes, GWAS SNPs are linked to gene expression using eQTL analysis. $\mathbf{b}$ To obtain greater understanding of disease pathogenesis, it is essential to look beyond the disruption of individual genes and identify potential disease-associated pathways. This can be done by identifying the co-expression relationships between genes in all loci linked to a specific disease, for example, using methods such as GRAIL [42], MAGENTA [43], and DEPICT [39]. c In the future, to pinpoint disease-relevant key driver genes, directional co-expression networks can be generated using a combination of current and novel approaches, including pseudotemporal ordering of scRNA-seq data and context-dependent eQTL and co-expression QTL analysis. eQTL expression quantitative trait locus, GWAS genome wide association studies, scRNA single-cell RNA, SNP single nucleotide polymorphism

\section{Implementing directionality in the gene network}

Disease-relevant gene clusters can be identified using the methods discussed above, but they do not provide insight into how genetic risk factors affect the network. To identify the downstream consequences of genetic risk factors, directionality must be added to co-expression networks. A GRN is a directional co-expression network that also has information about the regulators that control gene expression. Information obtained from databases such as KEGG [34] can be used to add directionality to specific pathways, but this information is limited in its ability to determine whether these pathways are active in specific cell types or if they function similarly in all cells or individuals. Additional approaches are therefore required to generate context-specific GRNs.

Directionality can be added to a co-expression network using a combination of perturbations, time-series data, and dynamic Bayesian models [44-46] (Fig. 2c; Box 1). However, dynamic Bayesian models cannot be made without time-series data, and generating such data is very costly because it requires a high sampling rate to correctly define directional relationships between genes (which follows from the Nyquist-Shannon sampling theorem that is used to find the sample frequency required to describe a continuous time signal $[47,48])$. Undersampling could result in incorrect correlations between genes or in missing key events in the network [49]. Moreover, correct directional inference requires cells to be synchronized to the same cell cycle state before time-series experiments are started, and even when successful, cells may lose their synchronization over time [50]. Finally, the construction of Bayesian networks is computationally intensive [51]. This burden can be reduced by including prior knowledge from other sources (e.g. pathway databases), but the quality of the resulting network will be determined by the reliability of that prior knowledge $[52,53]$.

Information about the regulators that control gene expression can also be gained by linking GWAS variants to 


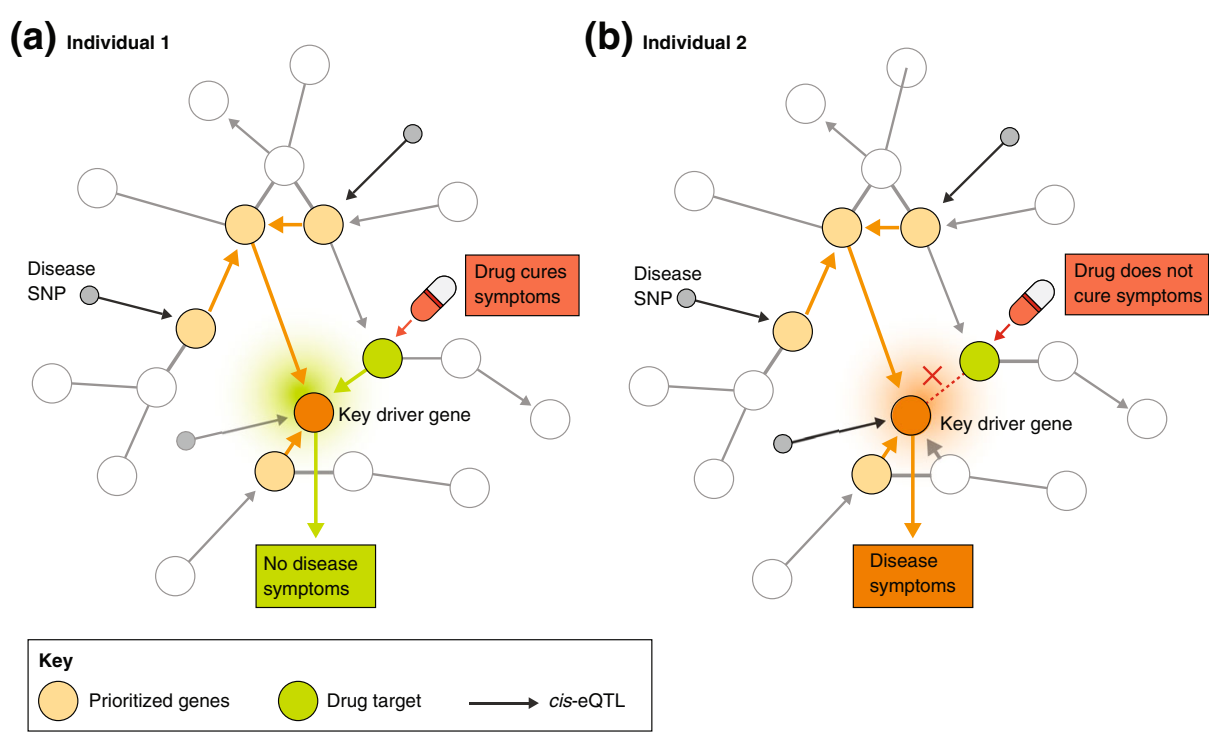

Fig. 3 Implications of personalized gene regulatory networks for precision medicine. Depending on an individual's regulatory wiring, specific drugs may or may not be effective. Personalized GRNs will provide guidance for precision medicine in the future. In this example, GRNs of two hypothetical patients are shown in which the regulatory wiring between the drug target gene and the key driver gene is different. a In individual 1, the drug target gene activates the key driver gene. $\mathbf{b}$ In individual 2, the interaction between both genes is absent. Thus, in individual 1 , the drug is effective, whereas in individual 2, the drug is ineffective. GRN gene regulatory network

additional molecular layers such as transcription factor binding motifs and enhancer and promoter elements [54]. This information can be helpful in determining directionality and understanding how genes are regulated, which provides additional support for putatively causal interactions. Similarly, eQTL information can be linked to additional molecular layers to reveal the functional mechanism of how the genotype affects interactions between genes, so-called context-dependent eQTLs (Fig. 4) [29].

Additional molecular data layers can be very informative for inferring directionality; however, these data are not always available in the disease-relevant context. Recent bulk-based RNA-seq studies have generated tissue-specific co-expression networks for up to 144 different tissues [55, 56]. However, the associated time and cost of implementing directionality and context-specificity have hampered the generation of tissue-specific GRNs in bulk data. In the following sections, we describe how a combination of bulk and single-cell data can be used to resolve these issues and to create GRNs that help us understand the link between genetic risk factors and disease.

\section{Improving networks with single-cell data}

The first single-cell RNA-sequencing (scRNA-seq) experiment was performed with a single cell in 2009 [57]. Since then, the technique has further developed and now more than a hundred thousand cells can be processed in parallel $[58,59]$. Recently, efforts have been made to build gene co-expression networks using scRNA-seq data [60-62]. The reliability of these networks improves with increasing numbers of cells, reads-per-gene, and genes-per-cell, but exact numbers are difficult to provide as they are influenced by many factors [61, 62]. We expect that such single-cell-based co-expression networks will be further improved when the consequences of low RNA capture efficiency are overcome [63-65]. One of these consequences is that many PCR cycles are required to generate sufficient material for sequencing, which can result in PCR amplification bias. To overcome this bias, unique molecular identifiers (UMIs; barcodes that tag unique RNA molecules) are added before PCR amplification [66]. However, the most important consequence of low RNA capture efficiency is the high number of dropouts. Dropouts are genes for which no transcript is captured, even though the cell expressed the mRNA. Gene expression imputation has been proposed as a solution for this problem (for a detailed comparison of recent imputation algorithms see [67]). Although several promising solutions have been developed, none have completely solved the problems surrounding the sparseness of single-cell data, and this will likely remain an area of intense study in the coming years.

Most aspects of reconstructing a co-expression network will not differ between single-cell and bulk expression data (reviewed in [33]). However, the assumptions underlying bulk-based network methods on the gene expression distribution (normal distribution) may not apply to single-cell expression data (zero-inflated negative binomial distribution) [68]. The unique features of single-cell data may provide opportunities to enhance the network and will 


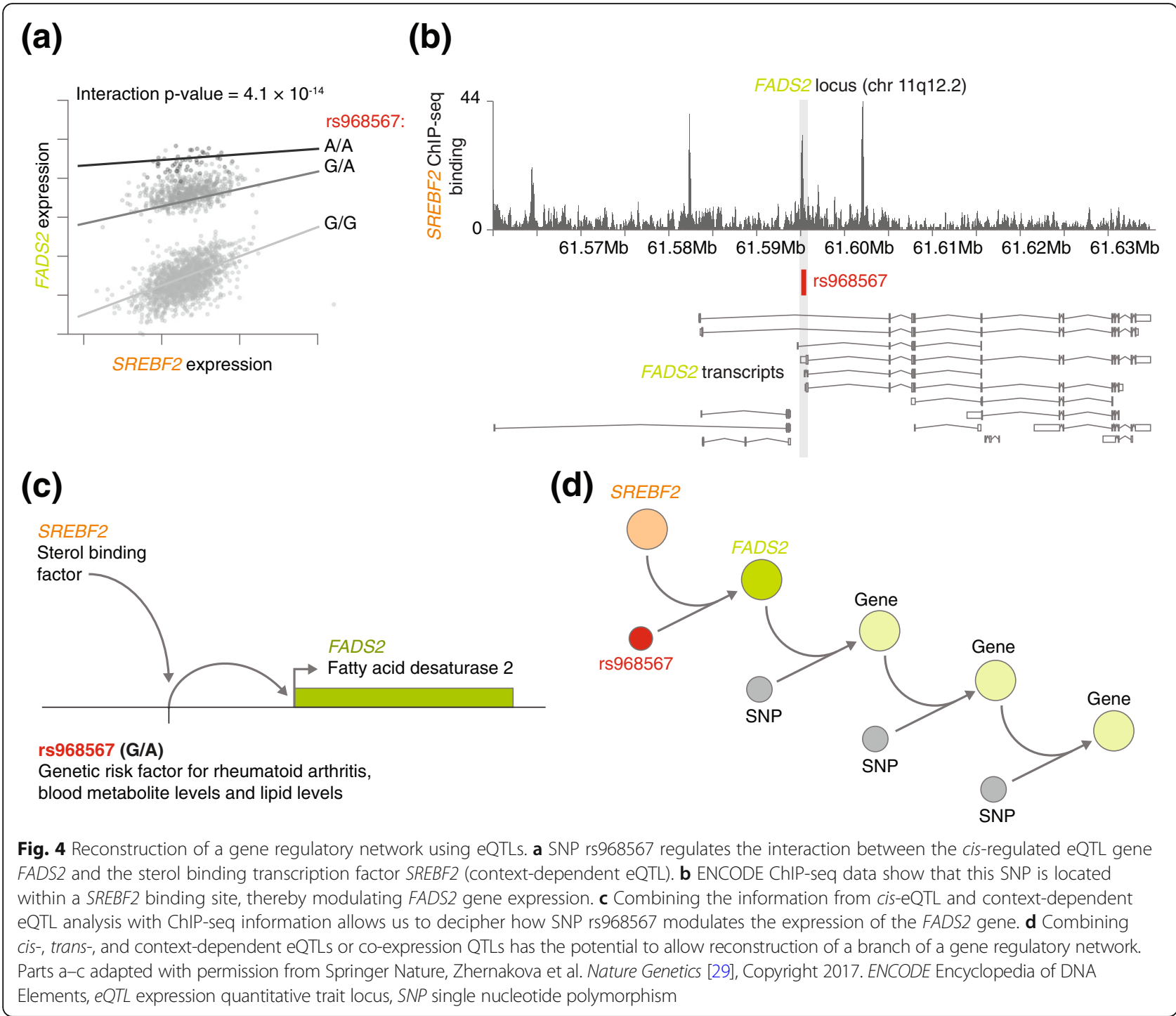

require the development of new algorithms to take these features into account. Ways to enhance the network using single-cell expression data are discussed in the following sections.

\section{Specifying the context}

Gene expression networks change depending on a number of factors, including cell type [22, 24, 25], environment [26, 27], and genetic signature [29, 30], and the influence of each of these contexts can be determined using scRNA-seq.

The ability of scRNA-seq data to dissect complex tissues and detect cell types/states in an unbiased manner [69-71] is valuable for reconstructing cell-type-specific co-expression networks. This kind of dissection using scRNA-seq was recently applied to detect single-cell eQTLs with high resolution [30, 72], which revealed that gene regulation can differ even between cell subtypes [30].
Unbiased classification has also led to the identification of specific cell states and combinations of transcription factors that drive cell-type-specific gene regulatory programs [73]. This study [73] showed that networks are different between brain cell types and that cell-type classification using networks gives better separation than classification based on gene expression levels alone.

Cellular heterogeneity induced by environmental perturbations can also be dissected using single-cell analysis [74]. In the context of co-expression networks, Martins et al. [75] used single-cell qRT-PCR to identify the heterogeneous effects of cytokine stimulations on the rewiring of the network in macrophages. Importantly, some of the effects on the co-expression network they identified would have been overlooked if they had pooled the expression of ten cells; a demonstration of how population-level co-expression networks cannot fully capture gene regulation at the single-cell level. 
scRNA-seq can also be used to identify differences induced by genetic variation between individuals, which enables the reconstruction of a person-specific or personalized co-expression network. In contrast to approaches using bulk RNA-seq, it is feasible to generate many measurements per individual with scRNA-seq, which enables the calculation of correlations between genes per individual [30]. These correlations can be used to identify the relationships between genes within a personal co-expression network. This approach was applied recently to identify relationships between genetic variants and the modulation of co-expression in $\mathrm{CD}_{4}^{+} \mathrm{T}$ cells. Within a cohort of 45 individuals, genetically modulated co-expression relationships, so-called co-expression QTLs, were identified that could be replicated in a bulk RNA-seq dataset of 2116 individuals. However, these relationships would not have been detected using a genome-wide approach in bulk data only [30]. Another advantage of scRNA-seq data is that true correlations between genes can be identified that would otherwise be masked by the effects of averaging in bulk RNA-seq data due to Simpson's paradox [76] (Box 1).

However, a disease-specific network is not defined by any of the above-mentioned factors (cell type, environment, or genetic signature) alone, but rather by a combination of them. Celiac disease, as an example, occurs only in individuals who carry specific HLA genotypes (genetics) and consume foods containing gluten (environment) [77]. Celiac disease is a well-known example of what is called a 'genotype by environment (GxE) interaction', where an environmental perturbation is modulated by an individual's genetic background. Future scRNA-seq studies should expand our understanding of how genotype by environment interactions modulate co-expression networks, for example, by exposing cells from different individuals to various environmental conditions.

\section{Exploiting scRNA-seq data variability to infer directionality} Measured gene expression levels can vary considerably between different cells even after accounting for cell type, environment, and genotype. The intercellular biological variability in gene expression levels provides unique insights that cannot easily be extracted from bulk expression data. During dynamic processes, such as cell differentiation or a response to an environmental stimulus, cells will move towards another state over time. However, the pace at which cells move into this new state differs between cells. By exploiting the asynchronous nature of these processes between cells, cells can be computationally ordered in pseudotime based on expression similarity $[78,79]$. This pseudotemporal ordering of cells can provide temporal resolution in an experiment that does not explicitly capture cells along a time-series. Insights can therefore be gained using scRNA-seq data that would remain hidden in bulk data, while requiring only one snapshot from a single sample (Fig. 5). At present, there are more than 50 different methods for pseudotemporal ordering of cells (see [80] for a recent comparison of these methods).

Pseudotime analyses have been used to reconstruct co-expression networks $[81,82]$ or small directional GRNs [83] from single-cell data (see [33] for an overview of current computational methods). However, the assumptions required for pseudotemporal ordering of cells are often violated in practice, which can result in incorrect assignment of directionality [84, 85]. The sampling frequency inferred by these methods, for instance, depends on sample size, which could be insufficient to recreate the complete underlying process of interest. Furthermore, several different networks may give plausible explanations for the same observed distribution of cell states. Therefore, it is difficult to determine the correct underlying mechanism of gene regulation without prior knowledge.

Both these issues can be resolved using a method called RNA velocity [86], which exploits the unidirectional character of splicing. RNA velocity examines the rate of change of mRNA molecule abundances in the cell by modeling the relationship between unspliced mRNA (an indicator of current transcription) and spliced mRNA (an indicator of transcription in the recent past). Although 3 '-end scRNA-seq data do not cover the entire mRNA transcript, these data as well as full-length data can be used to study RNA velocity. By taking the RNA velocity information of all genes together, a cell's future state can be successfully predicted $[86,87]$. Moreover, RNA velocity artificially enriches the inferred sampling frequency and prioritizes the pseudotemporal order that explains the observed distribution of cell states.

Interestingly, in the context of GRNs, combining the information extracted from RNA abundance and RNA velocity improves the ability to predict true targets of transcription factors across a range of species and for experimental settings that mimic the sparseness and noisiness of scRNA-seq data [88]. Moreover, a time-delay between gene-gene interactions can be implemented to reflect the delay in gene expression changes upon a gene-gene interaction. This was shown to result in greater accuracy to identify time-delayed interactions and infer network topology [89, 90]. As such, similar to application of time-series bulk data, we reason that causality can be inferred in GRNs using a combination of RNA velocity and pseudotemporal ordering (Fig. 5).

\section{Integrative approach for GRN assembly}

Considering the unique features and applicability of both bulk and scRNA-seq data for generating GRNs, we 
(a)
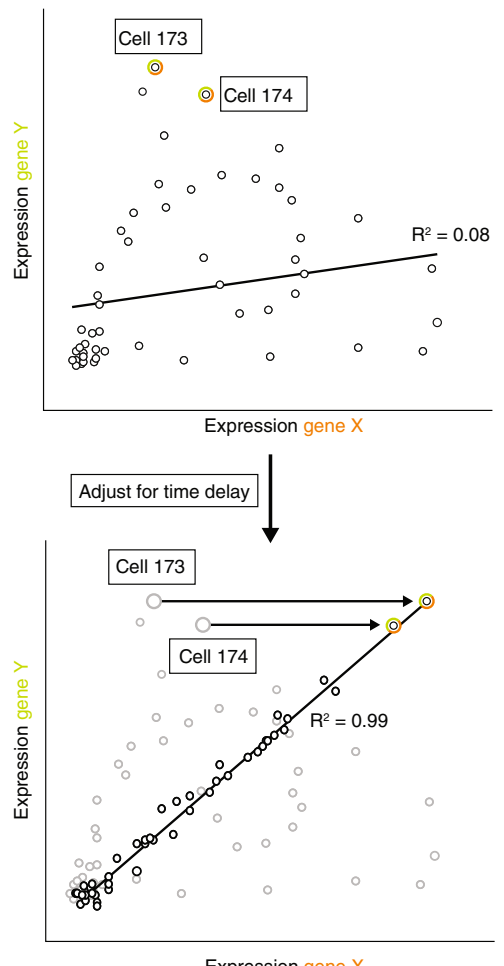

(b)
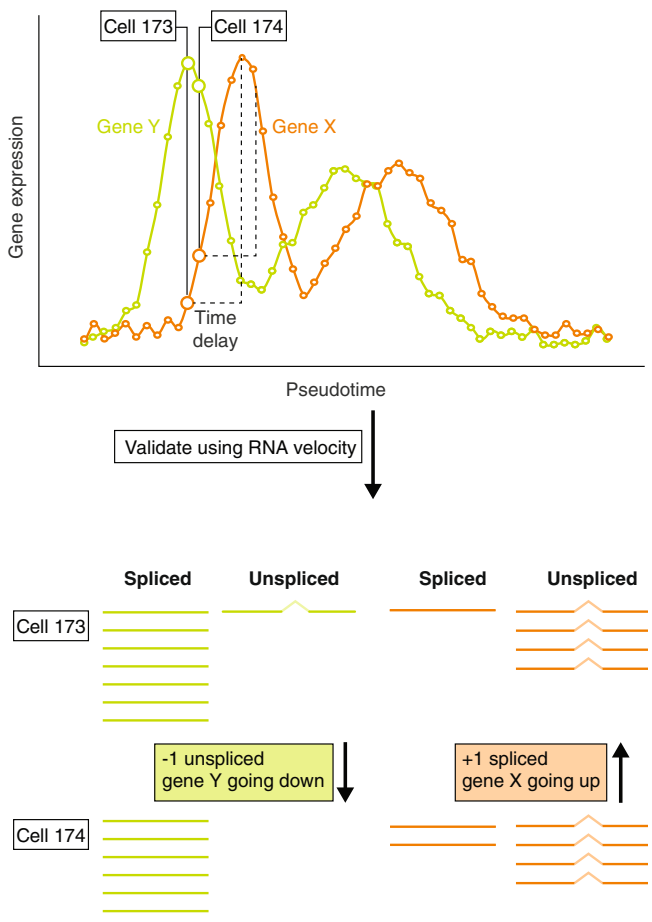

Fig. 5 Inferring causality using pseudotime analysis and RNA velocity. a In this hypothetical example, when determining the relationship between gene $X$ and gene $Y$, no correlation would be observed (top). However, the relationship between both genes may be masked by a time delay and correcting for this time delay might reveal a clear correlation between the expression of gene $X$ and gene $Y$ (bottom). $\mathbf{b}$ To identify the length of a time delay, the cells can be ordered along pseudotime, that is, an inferred timeline based on the variable gene expression states of single cells measured at a single moment in time (top). RNA velocity [86], a readout that exploits the unidirectional character of splicing, allows the prediction of the future state of a cell and its genes (bottom). As such, the correct ordering of cells can be validated using RNA velocity. Plotting gene expression against pseudotime shows that the expression of gene $X$ is following the expression of gene $Y$. From this, it can be deduced that gene $Y$ is regulating gene $X$, and not the other way around

propose using an integrative approach to assemble context-specific, personalized GRNs that can help move towards improved precision medicine in the future. This integrative approach combines the richness of bulk data with the finer detail and unique insights obtained from single cells (Fig. 6). Our proposed approach consists of an interplay alternating between bulk and single-cell data, iteratively updating GRNs with knowledge acquired from both sources of data. This allows us to take full advantage of both technologies and recent collaborative efforts, such as the Human Cell Atlas [91], the GTEx consortium [22], and the single-cell eQTLGen consortium [92]. In the following sections, we describe the three steps of this integrative approach using the example of hypothetical $\mathrm{CD}^{+}{ }^{+} \mathrm{T}$-cell data illustrated in Fig. 6.

\section{Bulk-based reference co-expression network}

The first step in assembling a context-specific GRN is establishing a cell-type-specific reference network that can be used as a baseline onto which the specific contexts can be projected. To create this reference network, numerous publicly available datasets for specific cell types made with bulk RNA-seq can be used. Public RNA-seq repositories, such as the European Nucleotide Archive [93] and the Sequence Read Archive [94], already contain hundreds of bulk RNA-seq datasets from purified cell types. Combining these datasets from different resources requires uniform alignment, quantification, and removal of batch effects [95], and several recent efforts have combined such uniformly processed bulk RNA-seq datasets in large repositories [96-99]. Based on previous benchmarking studies (comparing the performance of network reconstruction approaches against a known reference network) using both bulk and in silico data, community-based approaches seem most suitable for reconstructing such reference networks [100].

Although single-cell data provide a more detailed context of the network, at present they do not have the sensitivity of bulk data and will create an incomplete network 
From reference co-expression to causal gene regulatory networks

\section{Bulk gene expression data}

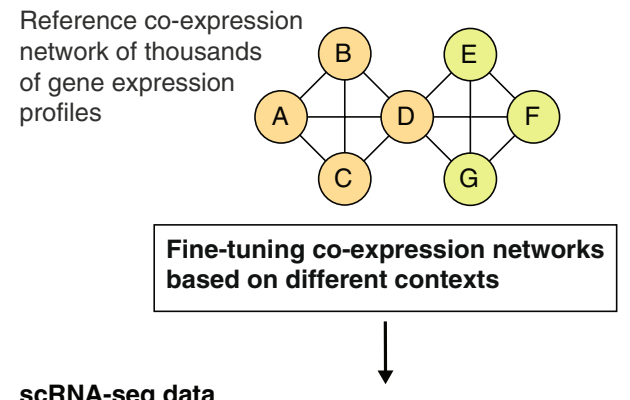

Cell types

- Cell type specificity

- Cell subtype specificity

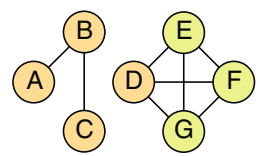

Network in $\mathrm{CD}^{+}{ }^{+} \mathrm{T}$ cells

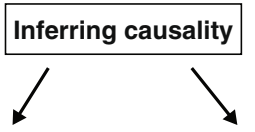

Bulk data

- Trans-eQTLs

- Context-specific eQTLs

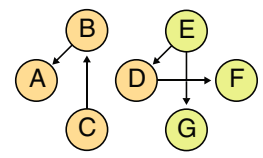

Causal gene regulatory network in $\mathrm{CD} 4^{+} \mathrm{T}$ cellls

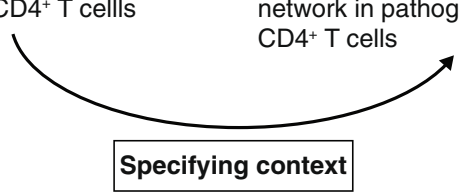

Fig. 6 Reconstruction of personalized, context-specific gene regulatory networks through the integration of bulk and single-cell data. We expect the richness of bulk expression data (for example, the number of genes or transcript variants detected and the number of datasets available for any given tissue) combined with the context-specificity of scRNA-seq data (for example, cell type and environmental exposure) will facilitate the generation of contextspecific co-expression networks. Finally, integrating additional data layers, such as context-specific eQTLs and co-expression QTLS combined with ChIP-seq data, will allow the direction of effects to be determined. This information will enable the reconstruction of personalized, context-specific gene regulatory networks for use in precision medicine due to dropouts. The bulk reference co-expression network thus serves two purposes. The first is to fill gaps in the network where expression, and therefore any possibility of an interaction, is missing for genes. The second is to provide additional supporting information when evidence from single-cell expression data is insufficient to confidently report the interaction between two genes. In this scenario, comparison between the single-cell and bulk RNA-seq reference can be used to gain additional support for the interaction.

To successfully use a bulk-based reference network, stable parts of the network, so-called anchor points, have to be identified. Anchor points are parts of the network that are shared between the reference network and the personalized single-cell network. With the bulk-based reference network as a basis on which the single-cell data can be projected, further context-specific connections can be investigated.

\section{Fine-tuning the reference co-expression network to reflect the context}

The second step in assembling the context-specific GRN is to use scRNA-seq data to add context-specific information to the bulk-based reference co-expression network. Single-cell data enable sampling to be performed on a whole tissue, after which individual cell types can be dissected using the single-cell expression profiles. This allows for the creation of cell-type-specific networks without the need to predefine the studied cell types. Furthermore, for each of the identified cell types, the effect of environmental perturbations can be studied. To illustrate this second step, we provide a hypothetical example in Fig. 6 of a CD4 ${ }^{+}$T-cell-specific and pathogen stimulation-perturbed network. By generating such a network for each individual separately, the higher complexity of the network can be captured.

Several single-cell-specific computational models have been developed to generate GRNs that could be used for this purpose [33]. Such models are required to correct for dropouts and to take the single-cell-specific gene expression distribution into account $[68,101]$. Nevertheless, benchmarking revealed that both general bulk-based and specific single-cell-based approaches showed poor performance using experimental and in silico single-cell data [68]. Benchmarking of these algorithms remains a challenge due to the lack of a gold standard network for comparison. The comparison network used at present is based on bulk data, and consists of interactions found in a combination of many cell types and contexts together resulting in a notable difference between bulk gold standard networks and networks derived from single-cell data [68]. This may be because interactions found in bulk-based reference networks are not truly representative of interactions found at the single-cell level. 
An experimentally validated single-cell gold standard network will advance the development of single-cellspecific network reconstruction algorithms. We believe that pooled CRISPR-screens coupled with a scRNA-seq readout, such as CROP-seq [102], CRISP-seq [103], and PERTURB-seq $[104,105]$, offer the possibility to create such a single-cell-based gold standard network. These methods enable mapping of the downstream consequences of gene perturbations on the whole transcriptome level. Not only can these experimental methods be used to create a gold standard, they can also provide insights into causal gene-gene relationships.

\section{Transitioning from associations to causal relationships}

The final step in assembling the GRN is to add directionality to the context-specific network to gain insight into the putatively causal relationships between genes and to validate them using experimental approaches. Our proposed method utilizes easily accessible data to solve the issue of directionality by integrating information from eQTLs or pseudotemporal ordering into the network.

There are several ways in which eQTLs can be used to gain insight into the GRN. First, they can reveal downstream effects of gene regulation. SNPs that have both cis and trans effects on gene expression can be used to uncover regulatory relationships between genes. For example, Westra et al. [24] have shown that such SNPs may affect the expression of a transcription factor in cis and consequently affect the expression of many downstream genes in trans. For a number of these downstream genes supporting ChIP-seq data were found, which suggest directionality of regulation. Second, context-dependent eQTLs [29] and co-expression QTLs [30] can uncover the upstream interactors of some genes and identify parts of the network where the relationships between genes change with genotype (Fig. 4). Altogether, by combining cis-, trans-, and context-dependent eQTLs or co-expression QTLs, branches of a GRN can be reconstructed and extended with genetic information.

To put the regulatory information obtained from eQTLs into the correct context, the cell types in which the eQTL effects manifest have to be identified [22, 24, 25]. Identification of trans-eQTLs and context-dependent eQTLs requires enormous sample sizes in bulk expression data (thousands of samples) to overcome a severe multipletesting burden $[24,29]$. Such massive datasets are currently only available for whole tissues in bulk (such as whole blood), but these do not allow identification of the relevant cell type. Although the sample size of single-cell datasets does not permit these analyses at the genome-wide level, single-cell datasets can be used to determine the cell type in which an eQTL effect identified from bulk data manifests. After pinpointing the relevant cell type, bulk multi-omics data of this specific cell type can be used to identify or verify the regulating mechanism behind the context-dependent interaction. For example, a genetic variant was shown to change enhancer-promoter looping by affecting the affinity of a cell-type-specific transcription factor [106]. By providing this kind of evidence for the regulating mechanism, causality can be integrated into the parts of the cell-type-specific GRN for which eQTLs can be found.

Combining pseudotemporal ordering with RNA velocity allows the identification of directionality between all genes, not just eQTL genes. Identifying which genes share similar expression patterns and the pseudotime at which they are expressed can establish the directional relationship between these genes (Fig. 5). van Dijk et al. [107] recently showed promising results with a comparable approach in which imputed gene expression scRNA-seq data were ordered along pseudotime. Subsequently, transcription factors and potential targets that change considerably along pseudotime were selected. In this way, they reconstructed a large GRN consisting of 719 transcriptional regulators and 11,126 downstream target genes. Of the predicted target genes that were tested, 92\% significantly overlapped with target genes as assessed by ATAC-seq (assay for transposase-accessible chromatin using sequencing). This study showed promising results to identify target genes without experimental perturbation. However, experimental validation is required to transition from associations to causal relationships. Our proposed integrative approach will help to provide focus on those regions of the network that are of particular interest and alleviates the need to perform experimental validation on every possible gene, thereby circumventing the high cost associated with testing all combinations. Altogether, we expect that such an integrative approach will enable the reconstruction of well-validated context-specific, personalized GRNs.

\section{The future of precision medicine}

A major challenge in healthcare today is that the majority of prescribed drugs are only effective in a small subset of patients [31]. This not only leads to money lost on ineffective drugs, but it also unnecessarily exposes patients to adverse drug side effects. Well-validated, context-specific, personalized GRNs will be essential to move from more traditional medicine towards precision medicine, which will provide treatment or preventive measures that will be effective for patients based on their specific genetic, environmental, and lifestyle characteristics (Fig. 3). In order to successfully implement the proposed ideas, several technical and practical challenges have to be overcome (Table 1). Overcoming these challenges will open the road for implementing GRNs for precision medicine.

Disease-specific GRNs may provide novel insights into disease pathogenesis and have enhanced power to 
Table 1 Challenges associated with implementation of the proposed integrative approach for precision medicine

\begin{tabular}{|c|c|c|c|}
\hline & Challenge & Solution & References \\
\hline \multirow[t]{4}{*}{ Technical challenges } & $\begin{array}{l}\text { Implementation of directionality and } \\
\text { causality }\end{array}$ & $\begin{array}{l}\text { eQTL, context-dependent eQTL and co-expression QTL } \\
\text { information } \\
\text { Time-series data and pseudotime combined with RNA } \\
\text { velocity } \\
\text { Experimental validation using CRISPR perturbations } \\
\text { coupled to scRNA-seq read-out (for example, CRISP-seq, } \\
\text { CROP-seq, and PERTURB-seq) }\end{array}$ & {$[24,29,30,86,102-105]$} \\
\hline & Dropouts & Gene expression and cross-omics imputation & {$[67,118,119]$} \\
\hline & Amplification bias & Unique molecular identifiers (UMIs) & [66] \\
\hline & $\begin{array}{l}\text { Combining single-cell data with a bulk } \\
\text { reference network }\end{array}$ & $\begin{array}{l}\text { Anchor points } \\
\text { Computational methods need to be developed }\end{array}$ & [120] \\
\hline \multirow[t]{4}{*}{ Practical challenges } & $\begin{array}{l}\text { Time and cost involved in collecting } \\
\text { scRNA-seq data }\end{array}$ & $\begin{array}{l}\text { Droplet-based approaches in combination with } \\
\text { approaches that enable super-loading and pooling of } \\
\text { samples (for example, cell hashing or demuxlet) } \\
\text { Split-pool barcoding approaches (for example, SPLiT- } \\
\text { seq and combinatorial indexing) } \\
\text { Large throughput sequencers that enable reduction in } \\
\text { sequencing cost }\end{array}$ & {$[58,59,72,121-124]$} \\
\hline & $\begin{array}{l}\text { Large-scale availability of datasets with } \\
\text { both genotype and scRNA-seq data }\end{array}$ & $\begin{array}{l}\text { Collaborative efforts (for example, single-cell eQTLGen } \\
\text { consortium and Human Cell Atlas) }\end{array}$ & {$[91,92]$} \\
\hline & $\begin{array}{l}\text { Cost involved in genotyping each } \\
\text { individual }\end{array}$ & $\begin{array}{l}\text { Genotype arrays in combination with imputation-based } \\
\text { approaches enable mapping of clinically relevant } \\
\text { genetic variants with high coverage for less than } € 100 \\
\text { per individual }\end{array}$ & {$[117,125,126]$} \\
\hline & Public perception, health regulations & $\begin{array}{l}\text { General Data Protection Regulation implemented in the } \\
\text { EU in } 2018 \\
\text { Genetic counselors to help with interpreting genetic } \\
\text { results }\end{array}$ & [113] \\
\hline
\end{tabular}

prioritize disease-causing genes [108]. These GRNs provide a bird's-eye view to look beyond the disruption of individual disease genes: each gene may have a small individual effect, but several disease genes together may have a large additive effect when converging into a few disrupted key regulatory pathways [109-111]. Despite the involvement of different individual disease genes, similar key regulatory pathways are likely to be disturbed in several different diseases. Likewise, exposure to specific environmental factors may disturb regulatory pathways in a fashion comparable to specific disease-associated genetic variants. These insights may provide novel links between different diseases or clues to how environmental factors can contribute to one or more diseases, and these new associations should provide novel directions for treatment.

Generation of context-specific GRNs may never fully capture the complexity of multifactorial interactions (for example, genetic background, environmental exposures, and disease) and the intercellular communication that influences the whole organism. Nevertheless, GRNs will be valuable for predicting the outcome of perturbations, and this particular function of GRNs will be useful for predicting potential drug targets for disease. Tumor-specific networks inferred using a combination of gene expression data and cancer-related signaling pathways have already been successfully applied to identify oncogenes and previously identified targets of cancer treatment [112].

An integral component for disease treatment based on a personalized GRN is to have a patient's genotype information available. Genotyping patients may allow doctors to select effective drugs while preventing unnecessary adverse effects for the patient. However, before this can be implemented in clinical practice, a shift in both public perception and healthcare regulations is required. For example, updated privacy and data protection regulations, such as the General Data Protection Regulation implemented in the EU in 2018 [113], will be important to reduce privacy concerns in the general public, as this puts individuals in control of their own data. With these recent developments in policy and public perception it is becoming more likely that more people will be genotyped, which will help to build personalized GRNs that can be used in precision medicine.

\section{Conclusions and future perspectives}

We have highlighted the importance of using a gene network-based approach rather than a single-gene focused approach to gain the bird's eye view required to understand disease pathogenesis. As diseases arise in highly specific contexts, context-dependent GRNs are needed to fully understand these diseases. To build these 
context-dependent GRNs, we have proposed an integrative approach of generating GRNs using both bulk and single-cell data. We have not described the computational implementation of our approach, as this would go beyond the scope of this article. Nevertheless, we expect that our iterative approach is well-suited to implementation using machine learning or deep learning models that learn from large datasets and make predictions on likely outcomes of complex cellular systems such as GRNs $[114,115]$. This requires generating massive datasets for which the first steps are being taken in consortia such as single-cell eQTLGen [92] and the Human Cell Atlas [91]. These datasets will be instrumental for executing our integrated approach using machine learning algorithms. Moreover, platforms such as the Human Cell Atlas are expected to provide more uniform guidelines and solutions for generating, processing, and handling large-scale scRNA-seq data. This will facilitate the combining of scRNA-seq datasets as part of our integrative approach.

As initiatives such as 23andMe [116] and the UK Biobank [117] produce ever larger genetic datasets that could be used to reconstruct personalized GRNs, and new initiatives are started, the ability to accurately predict disease risk through a combination of genotype associations and personalized GRNs will improve. However, before these personalized GRNs can be adopted in clinical practice, a number of ethical and legal issues will have to be resolved. Clinical guidelines, for instance, will have to be developed so that the interpretation of the results can be guided by trained professionals and the actionability of individual findings has to become clear [32]. Once these issues have been addressed, we expect that personalized, contextdependent GRNs will accelerate the progress required to make the next big leap in precision medicine.

\section{Abbreviations}

eQTL: Expression quantitative trait locus; GRN: Gene regulatory network; GTEx: Genotype-Tissue Expression project; GWAS: Genome-wide association study; GxE: Genotype by environment; scRNA-seq: Single-cell RNA-sequencing; SNP: Single nucleotide polymorphism; UMI: Unique molecular identifier

\section{Acknowledgements}

We thank J. Senior and K. Mclntyre for careful editing of the manuscript.

\section{Funding}

LF is supported by grants from the Dutch Research Council (ZonMW-VIDI 917.14.374) and a European Research Council Starting Grant, grant agreement 637640 (ImmRisk). The funding bodies did not have any role in the content or writing of this manuscript.

\section{Authors' contributions}

MW and DV defined the content and wrote the manuscript. All authors commented on and edited the text and approved the final version of the manuscript.

\section{Competing interests}

The authors declare that they have no competing interests.

\section{Publisher's Note}

Springer Nature remains neutral with regard to jurisdictional claims in published maps and institutional affiliations.

Published online: 19 December 2018

\section{References}

1. Visscher PM, Wray NR, Zhang Q, Sklar P, McCarthy Ml, Brown MA, et al. 10 years of GWAS discovery: biology, function, and translation. Am J Hum Genet. 2017;101(1):5-22.

2. Shi H, Kichaev G, Pasaniuc B. Contrasting the genetic architecture of 30 complex traits from summary association data. Am J Hum Genet. 2016;99(1): 139-53.

3. Yang J, Bakshi A, Zhu Z, Hemani G, Vinkhuyzen AA, Lee SH, et al. Genetic variance estimation with imputed variants finds negligible missing heritability for human height and body mass index. Nat Genet. 2015;47(10): 1114-20.

4. Brodie A, Azaria JR, Ofran Y. How far from the SNP may the causative genes be? Nucleic Acids Res. 2016;44(13):6046-54.

5. McGovern A, Schoenfelder S, Martin P, Massey J, Duffus K, Plant D, et al. Capture hi-C identifies a novel causal gene, IL20RA, in the pan-autoimmune genetic susceptibility region 6a23. Genome Biol. 2016;17(1):212.

6. Claussnitzer M, Dankel SN, Kim KH, Quon G, Meuleman W, Haugen C, et al. FTO obesity variant circuitry and adipocyte browning in humans. N Engl $\rfloor$ Med. 2015;373(10):895-907.

7. Smemo S, Tena JJ, Kim KH, Gamazon ER, Sakabe NJ, Gómez-Marín C, et al. Obesity-associated variants within FTO form long-range functional connections with IRX3. Nature. 2014;507(7492):371-5.

8. Schadt EE, Monks SA, Drake TA, Lusis AJ, Che N, Colinayo V, et al. Genetics of gene expression surveyed in maize, mouse and man. Nature. 2003; 422(6929):297-302.

9. Cheung VG, Conlin LK, Weber TM, Arcaro M, Jen KY, Morley M, et al. Natural variation in human gene expression assessed in lymphoblastoid cells. Nat Genet. 2003;33(3):422-5.

10. Zhu Z, Zhang F, Hu H, Bakshi A, Robinson MR, Powell JE, et al. Integration of summary data from GWAS and eQTL studies predicts complex trait gene targets. Nat Genet. 2016:48(5):481-7.

11. Schaid DJ, Chen W, Larson NB. From genome-wide associations to candidate causal variants by statistical fine-mapping. Nat Rev Genet. 2018; 19(8):491-504.

12. ENCODE Project Consortium. An integrated encyclopedia of DNA elements in the human genome. Nature. 2012;489(7414):57-74.

13. FANTOM Consortium and the RIKEN PMI and CLST (DGT), Forrest AR, Kawaji $\mathrm{H}$, Rehli M, Baillie JK, de Hoon MJ, et al. A promoter-level mammalian expression atlas. Nature. 2014;507(7493):462-70.

14. Consortium RE, Kundaje A, Meuleman W, Ernst J, Bilenky M, Yen A, et al. Integrative analysis of 111 reference human epigenomes. Nature. 2015; 518(7539):317-30.

15. Adams D, Altucci L, Antonarakis SE, Ballesteros J, Beck S, Bird A, et al. BLUEPRINT to decode the epigenetic signature written in blood. Nat Biotechnol. 2012;30(3):224-6.

16. Bell JT, Pai AA, Pickrell JK, Gaffney DJ, Pique-Regi R, Degner JF, et al. DNA methylation patterns associate with genetic and gene expression variation in HapMap cell lines. Genome Biol. 2011;12(1):R10.

17. McVicker G, van de Geijn B, Degner JF, Cain CE, Banovich NE, Raj A, et al. Identification of genetic variants that affect histone modifications in human cells. Science. 2013;342(6159):747-9.

18. Kasowski M, Grubert F, Heffelfinger C, Hariharan M, Asabere A, Waszak SM, et al. Variation in transcription factor binding among humans. Science. 2010 328(5975):232-5.

19. Degner JF, Pai AA, Pique-Regi R, Veyrieras JB, Gaffney DJ, Pickrell JK, et al. DNase I sensitivity QTLs are a major determinant of human expression variation. Nature. 2012;482(7385):390-4

20. Pai AA, Cain CE, Mizrahi-Man O, De Leon S, Lewellen N, Veyrieras JB, et al. The contribution of RNA decay quantitative trait loci to inter-individual variation in steady-state gene expression levels. PLoS Genet. 2012;8(10): e1003000.

21. Pickrell JK, Marioni JC, Pai AA, Degner JF, Engelhardt BE, Nkadori E, et al. Understanding mechanisms underlying human gene expression variation with RNA sequencing. Nature. 2010;464(7289):768-72. 
22. GTEx Consortium, Laboratory, Data Analysis \& Coordinating Center (LDACC)Analysis Working Group, Statistical Methods groups-Analysis Working Group, Enhancing GTEx (eGTEx) groups, NIH Common Fund, NIH/NCl, et al. Genetic effects on gene expression across human tissues. Nature. 2017:550(7675): 204-13.

23. Gamazon ER, Segrè AV, van de Bunt $M$, Wen $X, X i H S$, Hormozdiari F, et al. Using an atlas of gene regulation across 44 human tissues to inform complex disease- and trait-associated variation. Nat Genet. 2018:50(7):956-67.

24. Westra HJ, Peters MJ, Esko T, Yaghootkar H, Schurmann C, Kettunen J, et al. Systematic identification of trans eQTLs as putative drivers of known disease associations. Nat Genet. 2013:45(10):1238-43.

25. Battle A, Mostafavi S, Zhu X, Potash JB, Weissman MM, McCormick C, et al. Characterizing the genetic basis of transcriptome diversity through RNAsequencing of 922 individuals. Genome Res. 2014;24(1):14-24.

26. Knowles DA, Davis JR, Edgington H, Raj A, Fave MJ, Zhu X, et al. Allelespecific expression reveals interactions between genetic variation and environment. Nat Methods. 2017;14(7):699-702.

27. Favé MJ, Lamaze FC, Soave D, Hodgkinson A, Gauvin H, Bruat V, et al. Geneby-environment interactions in urban populations modulate risk phenotypes. Nat Commun. 2018;9(1):827.

28. Fagny M, Paulson JN, Kuijjer ML, Sonawane AR, Chen CY, Lopes-Ramos CM, et al. Exploring regulation in tissues with eQTL networks. Proc Natl Acad Sci U S A. 2017;114(37):E7841-50

29. Zhernakova DV, Deelen P, Vermaat M, van Iterson M, van Galen M, Arindrarto $W$, et al. Identification of context-dependent expression quantitative trait loci in whole blood. Nat Genet. 2017;49(1):139-45.

30. van der Wijst MGP, Brugge $H$, de Vries DH, Deelen P, Swertz MA, Franke L. Single-cell RNA sequencing identifies celltype-specific cis-eQTLs and coexpression QTLs. Nat Genet. 2018;50(4):493-7.

31. Schork NJ. Personalized medicine: time for one-person trials. Nature. 2015; 520(7549):609-11.

32. Karczewski KJ, Snyder MP. Integrative omics for health and disease. Nat Rev Genet. 2018;19(5):299-310.

33. Fiers MWEJ, Minnoye L, Aibar S, Bravo González-Blas C, Kalender Atak Z, Aerts S. Mapping gene regulatory networks from single-cell omics data. Brief Funct Genomics. 2018;17(4):246-54.

34. Ogata H, Goto S, Sato K, Fujibuchi W, Bono H, Kanehisa M. KEGG: Kyoto encyclopedia of genes and genomes. Nucleic Acids Res. 1999;27(1):29-34.

35. Fabregat A, Jupe S, Matthews L, Sidiropoulos K, Gillespie M, Garapati P, et al. The reactome pathway knowledgebase. Nucleic Acids Res. 2018;46(D1): D649-55.

36. van Dam S, Võsa U, van der Graaf A, Franke L, de Magalhães JP. Gene coexpression analysis for functional classification and gene-disease predictions. Brief Bioinform. 2018;19(4):575-92.

37. Wang $Y X$, Huang $H$. Review on statistical methods for gene network reconstruction using expression data. J Theor Biol. 2014;362:53-61.

38. Zhang B, Horvath S. A general framework for weighted gene co-expression network analysis. Stat Appl Genet Mol Biol. 2005;4:Article17.

39. Pers TH, Karjalainen JM, Chan Y, Westra HJ, Wood AR, Yang J, et al. Biological interpretation of genome-wide association studies using predicted gene functions. Nat Commun. 2015;6:5890

40. Saelens W, Cannoodt R, Saeys Y. A comprehensive evaluation of module detection methods for gene expression data. Nat Commun. 2018;9(1):1090.

41. Shim U, Kim HN, Lee H, Oh JY, Sung YA, Kim HL. Pathway analysis based on a genome-wide association study of polycystic ovary syndrome. PLoS One. 2015;10(8):e0136609.

42. Raychaudhuri S, Plenge RM, Rossin EJ, Ng AC, International Schizophrenia Consortium, Purcell SM, et al. Identifying relationships among genomic disease regions: predicting genes at pathogenic SNP associations and rare deletions. PLOS Genet. 2009;5(6):e1000534.

43. Segrè AV, DIAGRAM Consortium, MAGIC investigators, Groop L, Mootha VK, Daly MJ, et al. Common inherited variation in mitochondrial genes is not enriched for associations with type 2 diabetes or related glycemic traits. PLoS Genet. 2010;6(8). https://doi.org/10.1371/journal.pgen.1001058.

44. Sanchez-Castillo M, Blanco D, Tienda-Luna IM, Carrion MC, Huang Y. A Bayesian framework for the inference of gene regulatory networks from time and pseudo-time series data. Bioinformatics. 2018;34(6):964-70.

45. Sima C, Hua J, Jung S. Inference of gene regulatory networks using timeseries data: a survey. Curr Genomics. 2009;10(6):416-29.

46. Pe'er D, Regev A, Elidan G, Friedman N. Inferring subnetworks from perturbed expression profiles. Bioinformatics. 2001;17(Suppl 1):S215-24.
47. Whittaker ET. XVIII.-On the functions which are represented by the expansions of the interpolation-theory. Proc R Soc Edinburgh. 1915;35: 181-94.

48. Shannon CE. A mathematical theory of communication. Bell System Technical J. 1948;27(3):379-423.

49. Bar-Joseph Z. Analyzing time series gene expression data. Bioinformatics. 2004;20(16):2493-503.

50. Whitfield ML, Sherlock G, Saldanha AJ, Murray Jl, Ball CA, Alexander KE, et al. Identification of genes periodically expressed in the human cell cycle and their expression in tumors. Mol Biol Cell. 2002;13(6):1977-2000.

51. Tasaki S, Sauerwine B, Hoff B, Toyoshiba H, Gaiteri C, Chaibub NE. Bayesian network reconstruction using systems genetics data: comparison of MCMC methods. Genetics. 2015;199(4):973-89.

52. Liao W, Ji Q. Learning Bayesian network parameters under incomplete data with domain knowledge. Pattern Recogn. 2009;42(11):3046-56.

53. Feelders A, van der Gaag LC. Learning Bayesian network parameters with prior knowledge about context-specific qualitative influences. In: Bacchus F, Jaakkola T, editors. Proceedings of the twenty-first conference on uncertainty in artificial intelligence (UAI). Arlington, Virginia: AUAI Press; 2005. p. 193-200.

54. Marbach D, Lamparter D, Quon G, Kellis M, Kutalik Z, Bergmann S. Tissuespecific regulatory circuits reveal variable modular perturbations across complex diseases. Nat Methods. 2016;13(4):366-70.

55. Saha A, Kim Y, Gewirtz ADH, Jo B, Gao C, McDowell IC, et al. Co-expression networks reveal the tissue-specific regulation of transcription and splicing. Genome Res. 2017:27(11):1843-58.

56. Greene CS, Krishnan A, Wong AK, Ricciotti E, Zelaya RA, Himmelstein DS, et al. Understanding multicellular function and disease with human tissuespecific networks. Nat Genet. 2015;47(6):569-76.

57. Tang F, Barbacioru C, Wang Y, Nordman E, Lee C, Xu N, et al. mRNA-Seq whole-transcriptome analysis of a single cell. Nat Methods. 2009;6(5):377-82.

58. Rosenberg AB, Roco CM, Muscat RA, Kuchina A, Sample $P$, Yao Z, et al. Single-cell profiling of the developing mouse brain and spinal cord with split-pool barcoding. Science. 2018;360(6385):176-82.

59. Zheng GXY, Terry JM, Belgrader P, Ryvkin P, Bent ZW, Wilson R, et al. Massively parallel digital transcriptional profiling of single cells. Nat Commun. 2017:8:14049.

60. Herbach U, Bonnaffoux A, Espinasse T, Gandrillon O. Inferring gene regulatory networks from single-cell data: a mechanistic approach. BMC Syst Biol. 2017;11(1):105

61. Chan TE, Stumpf MPH, Babtie AC. Gene regulatory network inference from single-cell data using multivariate information measures. Cell Syst. 2017:5(3): 251-67.e3.

62. Bartlett TE, Müller S, Diaz A. Single-cell co-expression subnetwork analysis. Sci Rep. 2017:7(1):15066

63. Svensson V, Natarajan KN, Ly LH, Miragaia RJ, Labalette C, Macaulay IC, et al Power analysis of single-cell RNA-sequencing experiments. Nat Methods. 2017;14(4):381-7.

64. Zeisel A, Muñoz-Manchado AB, Codeluppi S, Lönnerberg P, La Manno G, Juréus $A$, et al. Brain structure. Cell types in the mouse cortex and hippocampus revealed by single-cell RNA-seq. Science. 2015;347(6226): $1138-42$.

65. Marinov GK, Williams BA, McCue K, Schroth GP, Gertz J, Myers RM, et al. From single-cell to cell-pool transcriptomes: stochasticity in gene expression and RNA splicing. Genome Res. 2014:24(3):496-510.

66. Islam S, Zeisel A, Joost S, La Manno G, Zajac P, Kasper M, et al. Quantitative single-cell RNA-seq with unique molecular identifiers. Nat Methods. 2014; 11(2):163-6.

67. Zhang $L$, Zhang S. Comparison of computational methods for imputing single-cell RNA-sequencing data. IEEE/ACM Trans Comput Biol Bioinform. 2018. https://doi.org/10.1109/TCBB.2018.2848633.

68. Chen S, Mar JC. Evaluating methods of inferring gene regulatory networks highlights their lack of performance for single cell gene expression data. BMC Bioinformatics. 2018;19(1):232.

69. Villani AC, Satija R, Reynolds G, Sarkizova S, Shekhar K, Fletcher J, et al. Single-cell RNA-seq reveals new types of human blood dendritic cells, monocytes, and progenitors. Science. 2017;356(6335). https://doi.org/10. 1126/science.aah4573.

70. Jaitin DA, Kenigsberg E, Keren-Shaul H, Elefant N, Paul F, Zaretsky I, et al. Massively parallel single-cell RNA-seq for marker-free decomposition of tissues into cell types. Science. 2014;343(6172):776-9. 
71. Buettner F, Natarajan KN, Casale FP, Proserpio V, Scialdone A, Theis FJ, et al. Computational analysis of cell-to-cell heterogeneity in single-cell RNAsequencing data reveals hidden subpopulations of cells. Nat Biotechnol. 2015;33(2):155-60.

72. Kang HM, Subramaniam M, Targ S, Nguyen M, Maliskova L, McCarthy E, et al. Multiplexed droplet single-cell RNA-sequencing using natural genetic variation. Nat Biotechnol. 2017;36(1):89-94.

73. Aibar S, González-Blas CB, Moerman T, Huynh-Thu VA, Imrichova H, Hulselmans G, et al. SCENIC: single-cell regulatory network inference and clustering. Nat Methods. 2017;14(11):1083-6.

74. Butler A, Hoffman P, Smibert P, Papalexi E, Satija R. Integrating single-cell transcriptomic data across different conditions, technologies, and species. Nat Biotechnol. 2018;36(5):411-20.

75. Martins AJ, Narayanan M, Prüstel T, Fixsen B, Park K, Gottschalk RA, et al. Environment tunes propagation of cell-to-cell variation in the human macrophage gene network. Cell Syst. 2017;4(4):379-92.e12.

76. Simpson EH. The interpretation of interaction in contingency tables. J R Stat Soc Series B Methodol. 1951;13(2):238-41.

77. Sollid LM, Jabri B. Triggers and drivers of autoimmunity: lessons from coeliac disease. Nat Rev Immunol. 2013;13(4):294-302.

78. Ji Z, Ji H. TSCAN: pseudo-time reconstruction and evaluation in single-cell RNA-seq analysis. Nucleic Acids Res. 2016;44(13):e117.

79. Trapnell C, Cacchiarelli D, Grimsby J, Pokharel P, Li S, Morse M, et al. The dynamics and regulators of cell fate decisions are revealed by pseudotemporal ordering of single cells. Nat Biotechnol. 2014;32(4):381-6.

80. Saelens W, Cannoodt R, Todorov H, Saeys Y. A comparison of single-cell trajectory inference methods: towards more accurate and robust tools. bioRxiv. 2018; https://doi.org/10.1101/276907.

81. Specht AT, Li J. LEAP: constructing gene co-expression networks for singlecell RNA-sequencing data using pseudotime ordering. Bioinformatics. 2017; 33(5):764-6

82. Hamey FK, Nestorowa S, Kinston SJ, Kent DG, Wilson NK, Göttgens B. Reconstructing blood stem cell regulatory network models from single-cell molecular profiles. Proc Natl Acad Sci U S A. 2017;114(23):5822-9.

83. Ocone A, Haghverdi L, Mueller NS, Theis FJ. Reconstructing gene regulatory dynamics from high-dimensional single-cell snapshot data. Bioinformatics. 2015;31(12):i89-96

84. Weinreb C, Wolock S, Tusi BK, Socolovsky M, Klein AM. Fundamental limits on dynamic inference from single-cell snapshots. Proc Natl Acad Sci U S A. 2018;115(10):E2467-76.

85. Rashid S, Kotton DN, Bar-Joseph Z. TASIC: determining branching models from time series single cell data. Bioinformatics. 2017;33(16):2504-12.

86. La Manno G, Soldatov R, Zeisel A, Braun E, Hochgerner H, Petukhov V, et al. RNA velocity of single cells. Nature. 2018;560(7719):494-8.

87. Plass M, Solana J, Wolf FA, Ayoub S, Misios A, Glažar P, et al. Cell type atlas and lineage tree of a whole complex animal by single-cell transcriptomics. Science. 2018. https://doi.org/10.1126/science.aaq1723.

88. Desai JS, Sartor RC, Lawas LM, Jagadish SVK, Doherty CJ. Improving gene regulatory network inference by incorporating rates of transcriptional changes. Sci Rep. 2017;7(1):17244

89. Finkle JD, Wu JJ, Bagheri N. Windowed granger causal inference strategy improves discovery of gene regulatory networks. Proc Natl Acad Sci U S A. 2018;115(9):2252-7.

90. Schmitt WA Jr, Raab RM, Stephanopoulos G. Elucidation of gene interaction networks through time-lagged correlation analysis of transcriptional data. Genome Res. 2004;14(8):1654-63.

91. Regev A, Teichmann SA, Lander ES, Amit I, Benoist C, Birney E, et al. The human cell atlas. elife. 2017;6. https://doi.org/10.7554/eLife.27041.

92. Single-cell eQTLGen. http://eqtlgen.org/single-cell. Accessed 16 Nov 2018.

93. Silvester N, Alako B, Amid C, Cerdeño-Tarrága A, Clarke L, Cleland I, et al. The European nucleotide archive in 2017. Nucleic Acids Res. 2018;46(D1): D36-40.

94. Leinonen R, Sugawara H, Shumway M. International Nucleotide Sequence Database Collaboration The sequence read archive. Nucleic Acids Res. 2011; 39:D19-21.

95. Wang Q, Armenia J, Zhang C, Penson AV, Reznik E, Zhang L, et al. Unifying cancer and normal RNA sequencing data from different sources. Sci Data. 2018;5:180061

96. Li JR, Sun CH, Li W, Chao RF, Huang CC, Zhou XJ, et al. Cancer RNA-Seq Nexus: a database of phenotype-specific transcriptome profiling in cancer cells. Nucleic Acids Res. 2016;44(D1):D944-51.
97. Vivian J, Rao AA, Nothaft FA, Ketchum C, Armstrong J, Novak A, et al. Toil enables reproducible, open source, big biomedical data analyses. Nat Biotechnol. 2017;35(4):314-6.

98. Collado-Torres L, Nellore A, Kammers K, Ellis SE, Taub MA, Hansen KD, et al. Reproducible RNA-seq analysis using recount2. Nat Biotechnol. 2017;35(4): 319-21.

99. Lachmann A, Torre D, Keenan AB, Jagodnik KM, Lee HJ, Wang L, et al. Massive mining of publicly available RNA-seq data from human and mouse. Nat Commun. 2018;9(1):1366

100. Marbach D, Costello JC, Küffner R, Vega NM, Prill RJ, Camacho DM, et al. Wisdom of crowds for robust gene network inference. Nat Methods. 2012; 9(8):796-804.

101. Ghazanfar S, Bisogni AJ, Ormerod JT, Lin DM, Yang JY. Integrated single cell data analysis reveals cell specific networks and novel coactivation markers. BMC Syst Biol. 2016;10(Suppl 5):127.

102. Datlinger $P$, Rendeiro AF, Schmidl C, Krausgruber T, Traxler $P$, Klughammer J, et al. Pooled CRISPR screening with single-cell transcriptome readout. Nat Methods. 2017;14(3):297-301.

103. Jaitin DA, Weiner A, Yofe I, Lara-Astiaso D, Keren-Shaul H, David E, et al. Dissecting immune circuits by linking CRISPR-pooled screens with singlecell RNA-seq. Cell. 2016;167(7):1883-96.e15.

104. Dixit A, Parnas O, Li B, Chen J, Fulco CP, Jerby-Arnon L, et al. Perturb-Seq: dissecting molecular circuits with scalable single-cell RNA profiling of pooled genetic screens. Cell. 2016;167(7):1853-66.e17.

105. Adamson B, Norman TM, Jost M, Cho MY, Nuñez JK, Chen Y, et al. A multiplexed single-cell CRISPR Screening platform enables systematic dissection of the unfolded protein response. Cell. 2016;167(7):1867-82.e21.

106. Weintraub AS, Li CH, Zamudio AV, Sigova AA, Hannett NM, Day DS, et al. YY1 is a structural regulator of enhancer-promoter loops. Cell. 2017;171(7): 1573-88.e28.

107. van Dijk D, Sharma R, Nainys J, Yim K, Kathail P, Carr AJ, et al. Recovering gene interactions from single-cell data using data diffusion. Cell. 2018; 174(3):716-29.e27

108. Gao L, Uzun Y, Gao P, He B, Ma X, Wang J, et al. Identifying noncoding risk variants using disease-relevant gene regulatory networks. Nat Commun. 2018;9(1):702.

109. Menche J, Guney E, Sharma A, Branigan PJ, Loza MJ, Baribaud F, et al. Integrating personalized gene expression profiles into predictive diseaseassociated gene pools. NPJ Syst Biol Appl. 2017;3:10.

110. Menche J, Sharma A, Kitsak M, Ghiassian SD, Vidal M, Loscalzo J, et al. Disease networks. Uncovering disease-disease relationships through the incomplete interactome. Science. 2015;347(6224):1257601.

111. Chatterjee S, Kapoor A, Akiyama JA, Auer DR, Lee D, Gabriel S, et al. Enhancer variants synergistically drive dysfunction of a gene regulatory network in Hirschsprung disease. Cell. 2016;167(2):355-68.e10.

112. Peng Q, Schork NJ. Utility of network integrity methods in therapeutic target identification. Front Genet. 2014;5:12. https://doi.org/10.3389/fgene. 2014.00012

113. European Union. Regulation (EU) 2016/679 of the European Parliament and of the council of 27 April 2016 on the protection of natural persons with regard to the processing of personal data and on the free movement of such data, and repealing directive 95/46/EC (general data protection regulation). Official J Eur Union. 2016;L119:1-88.

114. Camacho DM, Collins KM, Powers RK, Costello JC, Collins JJ. Next-generation machine learning for biological networks. Cell. 2018;173(7):1581-92.

115. Ching T, Himmelstein DS, Beaulieu-Jones BK, Kalinin AA, Do BT, Way GP, et al. Opportunities and obstacles for deep learning in biology and medicine. J R Soc Interface. 2018;15(141). https://doi.org/10.1098/rsif.2017.0387.

116. Stoeklé HC, Mamzer-Bruneel MF, Vogt G, Hervé C. 23andMe: a new twosided data-banking market model. BMC Med Ethics. 2016;17:19. https://doi. org/10.1186/s12910-016-0101-9.

117. Bycroft C, Freeman C, Petkova D, Band G, Elliott LT, Sharp K, et al. Genomewide genetic data on $\sim 500,000$ UK Biobank participants. bioRxiv. 2017; doi: https://doi.org/10.1101/166298.

118. Buenrostro JD, Corces MR, Lareau CA, Wu B, Schep AN, Aryee MJ, et al. Integrated single-cell analysis maps the continuous regulatory landscape of human hematopoietic differentiation. Cell. 2018;173(6):1535-48.e16.

119. Welch JD, Hartemink AJ, Prins JF. MATCHER: manifold alignment reveals correspondence between single cell transcriptome and epigenome dynamics. Genome Biol. 2017;18(1):138. https://doi.org/10.1186/s13059-017$1269-0$ 
120. Stuart T, Butler A, Hoffman P, Hafemeister C, Papalexi E, Mauck WM, et al. Comprehensive integration of single cell data. bioRxiv. 2018; https://doi.org/ 10.1101/460147.

121. Cao J, Packer JS, Ramani V, Cusanovich DA, Huynh C, Daza R, et al. Comprehensive single-cell transcriptional profiling of a multicellular organism. Science. 2017;357(6352):661-7.

122. Stoeckius M, Hafemeister C, Stephenson W, Houck-Loomis B, Chattopadhyay PK, Swerdlow $H$, et al. Simultaneous epitope and transcriptome measurement in single cells. Nat Methods. 2017;14(9):865-8.

123. Macosko EZ, Basu A, Satija R, Nemesh J, Shekhar K, Goldman M, et al. Highly parallel genome-wide expression profiling of individual cells using nanoliter droplets. Cell. 2015;161(5):1202-14.

124. Stoeckius M, Zheng S, Houck-Loomis B, Hao S, Yeung B, Smibert P, et al. Cell "hashing" with barcoded antibodies enables multiplexing and doublet detection for single cell genomics. bioRxiv. 2017; doi: https://doi.org/10. $1101 / 237693$.

125. Illumina. Infinium global screening array-24 kit (1152 samples). https://www. illumina.com/products/by-type/microarray-kits/infinium-global-screening html. Accessed 3 Dec 2018.

126. Marchini J, Howie B. Genotype imputation for genome-wide association studies. Nat Rev Genet. 2010;11(7):499-511. 